\title{
Rare Anemias: Are Their Names Just Smoke and Mirrors?
}

\author{
Greta Simionato $^{1,2}$, Richard van Wijk ${ }^{3}$, Stephan Quint ${ }^{2,4}$, Christian Wagner ${ }^{2,5}$, \\ Paola Bianchi ${ }^{6}$ and Lars Kaestner ${ }^{2,7 *}$ \\ ${ }^{1}$ Institute for Clinical and Experimental Surgery, Campus University Hospital, Saarland University, Homburg, Germany, \\ ${ }^{2}$ Experimental Physics, Dynamics of Fluids Group, Saarland University, Saarbrücken, Germany, ${ }^{3}$ Central Diagnostic \\ Laboratory - Research, University Medical Center Utrecht, Utrecht University, Utrecht, Netherlands, ${ }^{4} \mathrm{Cysmic}$ GmbH, \\ Saarbrücken, Germany, ${ }^{5}$ Physics and Materials Science Research Unit, University of Luxembourg, Luxembourg, \\ Luxembourg, ${ }^{6}$ Fondazione Instituto di Ricovero e Cura a Carattere Scientifico Ca' Granda Ospedale Maggiore Policlinico \\ Milano, Unità Operativa Complessa Ematologia, Unità Operativa Semplice Fisiopatologia delle Anemie, Milan, Italy, \\ ${ }^{7}$ Theoretical Medicine and Biosciences, Campus University Hospital, Saarland University, Homburg, Germany
}

Keywords: rare anemias, red cell morphology, hereditary spherocytosis, dehydrated stomatocytosis, hereditary xerocytosis, Gárdos channelopathy

\section{INTRODUCTION}

Numerous anemias and even neurodegenerative diseases are named after the predominant red blood cell (RBC) shape observed by microscopy - within this paper we refer to this as RBC morphology. Examples are spherocytes in hereditary spherocytosis (Huisjes et al., 2019), stomatocytes in hereditary stomatocytosis (Andolfo et al., 2018), elliptocytes in hereditary elliptocytosis (Soderquist and Bagg, 2013), sickle shaped deoxygenated RBCs in sickle cell disease (Cisneros and Thein, 2020), or acanthocytes in neuroacanthocytosis syndromes (Peikert et al., 2017).

A good portion of these names are justified because a substantial portion of the patient RBCs show at least under particular conditions the corresponding morphology. At the same time we have to admit that sometimes the shape classification can be confusing when, for example, sphero-ovalocytes (Jarolim et al., 1995), ovalocytes (Mohandas et al., 1984), elliptocytes (Motulsky et al., 1954), or poikilocytes (Agre et al., 1981) can all be attributed to the same disease: elliptocytosis.

The clinical evaluation of the cell shapes depends widely on the use of peripheral blood smears, i.e., a drop of fresh blood is smeared on a glass slide, dried, fixed and stained, a procedure that partly deteriorates the original cell morphology (Wenk, 1976). Extensive work was performed to characterize RBC shapes at electron microscopic super-resolution in 3D. These partly artistic images culminated in the seminal work by Marcel Bessis in the last century (Bessis, 1973). In the meantime, optical imaging technology progressed, e.g., confocal microscopy became widely available (Pawley, 2006) and was explored for the 3D-visualization of RBCs both in stasis (Khairy et al., 2008) and in flow (Quint et al., 2017). Optical technologies have less stringent requirements for sample preparation compared to electron microscopy (Abay et al., 2019). Confocal microscopy combined with automated processes including machine learning-based algorithms seems to lead to a revival of $\mathrm{RBC}$ visualization in $3 \mathrm{D}$ and the subsequent shape evaluation (Kaestner and Bianchi, 2020), shedding new lights on RBC morphological complexity. It is worthwhile to mention that $3 \mathrm{D}$ renderings based on confocal microscopy allow, in contrast to electron microscopy and probe scanning techniques, an unlimited $360^{\circ}$ view.

Furthermore, RBC in their physiological environment are in constant flow and at least in capillary flow take completely different cell shapes as in stasis, e.g., discocytes are transformed into "croissants" or "slippers" in a flow speed dependent manner (Kihm et al., 2018). For most pathophysiologial conditions RBC morphology in (capillary) flow are not investigated, which is a so far missed diagnostic potential but at the same time is not in favor of naming anemias after RBC shapes in stasis. 
Here we like to discuss examples of rare anemias named after the RBC shapes found in blood smears that are not representative of the disease condition based on both new microscopy applications and functional tests.

\section{SPHEROCYTE NUMBERS IN HEREDITARY SPHEROCYTOSIS - COMPARISON BETWEEN 2D AND 3D IMAGES}

A recent study investigated the use of an artificial neural network to automatically recognize $\mathrm{RBC}$ shapes, proposing that the detailed 3D analysis may even identify the specific genetic defect causing a particular rare anemia (Simionato et al., 2021). As a side information, it was found that the spherocytes identified in peripheral blood smears from patients with hereditary spherocytosis are mostly just "pseudo spherocytes" (see Figure 1). The figure illustrates that information indeed gets lost in 2D images. Furthermore, the almost exclusive occurrence of "pseudo spherocytes" per se (not their number) seems to be independent of the particular mutation causing the hereditary spherocytosis.

This finding is actually not new, since Bessis already stated: "The cells it describes (spherocytes) are actually not spheres. They include a variety of cells which are etiologically and morphologically dissimilar. They have only one thing in common: an increase in their thickness" (Bessis, 1974). The scanning electron microscopy (SEM) allows a 3D surface scan (although not a $360^{\circ}$ view), which allowed Bessis to make this statement and which is $100 \%$ confirmed by the $3 \mathrm{D}$ renderings based on confocal measurements (Figure 1).

\section{DEHYDRATED STOMATOCYTOSIS IS A TERMINOLOGY CONTRADICTION IN ITSELF}

When referring to $\mathrm{RBC}$ dehydration, currently we face two completely different approaches. The traditional one refers to the RBC shapes. If cells dehydrate, i.e., if they lose water, they shrink and this is associated with the formation of echinocytes. This holds true albeit there are also other isovolumetric mechanisms to transform discocytes into echinocytes (Fischer, 2003). The well-known but mechanistically elusive "glass effect" belongs to this kind of transformation. The way around, if $\mathrm{RBC}$ overhydrate, the uptake of water swells the discocytes toward a sphere through intermediate forms of stomatocytes (Lim et al., 2002). From that point of view, the terminology of "dehydrated stomatocytosis" is indeed a contradiction in itself. The alternative denomination for "dehydrated stomatocytosis" is "xerocytosis," indicating the occurrence of another pathophysiological cell shape, the xerocytes. This partly addresses the aforementioned contradiction but the alternative use of both terms, "dehydrated stomatocytosis" and "xerocytosis" adds to the confusion.

However, there is the concept of testing osmotic resistance with the ektacytometry as an incarnation that allows widely automated testing under reproducible and interlaboratory comparable conditions (Bianchi et al., 2015). This method became a kind of clinical standard and proved its usefulness in numerous hematological centers (Lazarova et al., 2017; LlaudetPlanas et al., 2018; Zaninoni et al., 2018; Vives-Corrons et al., 2021). In ektacytometry, a left shift of the osmotic fragility curve is compatible with a dehydration of the RBCs and due to differences in this curve, stomatocytosis was categorized into dehydrated and overhydrated stomatocytosis.

When comparing the two approaches, we favor the traditional cell shape-based concept because there is hardly any other option to explain the formation of stomatocytes, than overhydration. In contrast, a left shift of the curve in ektacytometry could have numerous explanations including the versatile composition of transmembrane transport proteins involved in volume regulation, such as the ion pumps $\mathrm{Na}^{+} / \mathrm{K}^{+}$-ATPase (Petrushanko et al., 2017), the $\mathrm{Ca}^{2+}$-ATPase (Dagher and Lew, 1988), ion channels such as TRPC6, Piezo1, the Gardos channel (Kaestner et al., 2020) or the recently described TRPV2 (Belkacemi et al., 2021), membrane transporters like band 3 protein (Bamberg and Passow, 1992) or the $\mathrm{K}^{+} / \mathrm{Cl}^{-}$-cotransporter (Adragna et al., 2006). Just to make it clear, we believe in the usefulness of ektacytometry as a diagnostic parameter for RBC-related diseases. It is only the strict terminological link to the hydration state that we like to discuss.

Although there is a deterministic relationship between hydration state and cell morphology in healthy RBCs as indicated by the SDE-scale (Lim et al., 2002), in the pathophysiological situations this relationship is widely distorted as the dehydration due to hemoglobinopathies indicate (Zaninoni et al., 2018; Krishnevskaya et al., 2021). Therefore, we believe the hydration state of the RBCs is an unsuitable parameter for disease classification.

\section{DISCUSSION}

The explanations above outline that a part of the nomenclature for rare anemias is not quite appropriate. The intention of this opinion paper is not to come up with a new terminology but to initiate the discussion about it. One possible and timely opportunity would be to orient the nomenclature of hereditary anemias on the mutation they are caused by, as it is done in other diseases, such as the "VSP13A disease," which was proposed to replace "Chorea Acanthocytosis" (Walker and Danek, 2021). In hereditary dehydrated stomatocytosis/hereditary xerocytosis, we have partly experienced such a change. Mutations of the mechanosensitive ion channel Piezol were initially identified as the molecular cause for this disease (Zarychanski et al., 2012; Albuisson et al., 2013; Andolfo et al., 2013; Shmukler et al., 2014; Rotordam et al., 2018). Soon afterwards, a second molecular player was identified, namely the Gárdos channel (Andolfo et al., 2015; Glogowska et al., 2015; Rapetti-Mauss et al., 2015). However, it is increasingly recognized that the mutations of the Gárdos channel result in a distinct pathological phenotype compared to the mutations of Piezol and the former is now referred to as Gárdos channelopathy or KCNN4 variants (Fermo et al., 2017, 2020). Ever since there is a kind of naming 


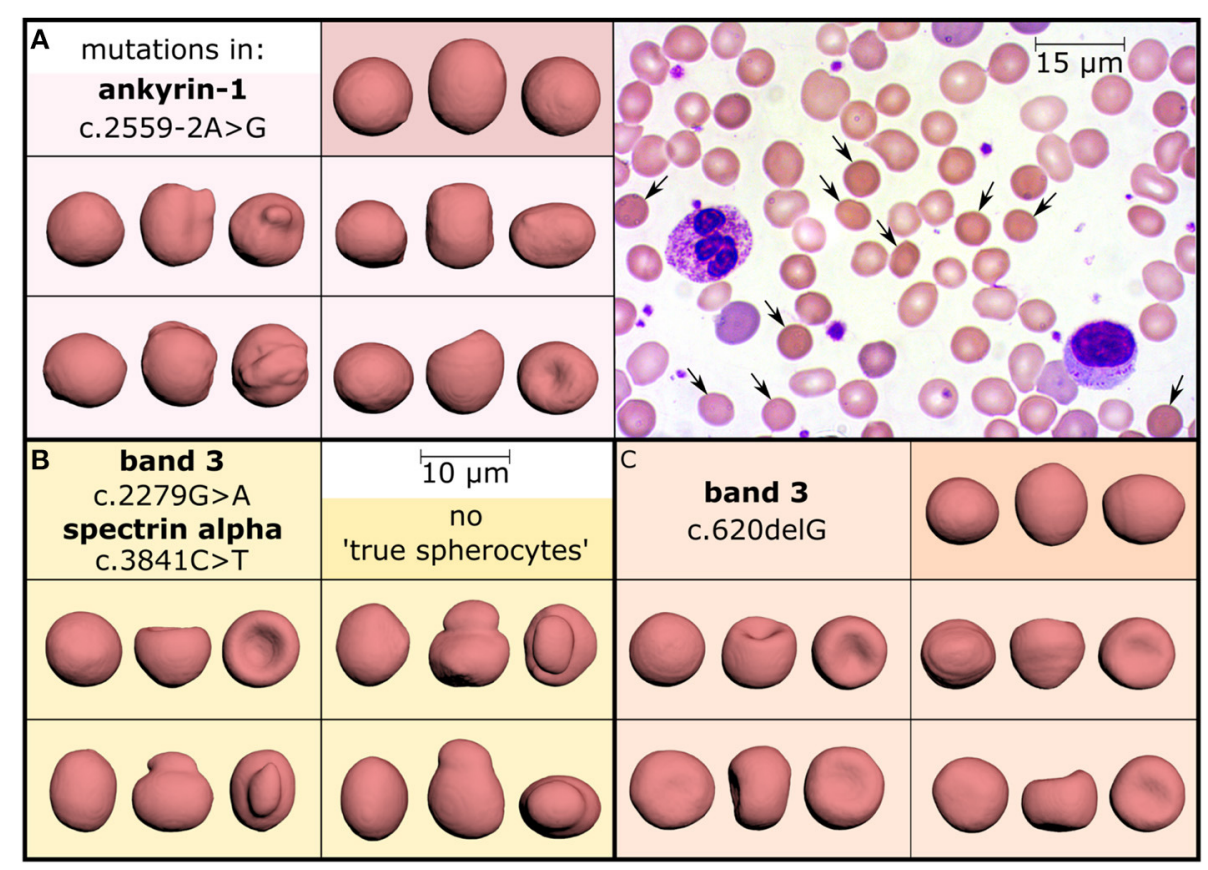

FIGURE 1 | Investigation of hereditary spherocytosis red blood cell shapes. Three patients diagnosed with hereditary spherocytosis caused by different mutations (panels A-C) showed a spherocyte count of 11\% (A), $8 \%$ (B), and 10\% (C) in their stained peripheral blood smears, as exemplified in panel A (arrows, objective-magnification 100x). Comparison with 3D-rendered confocal recordings (objective-magnification 60x) of glutaraldehyde fixed and CellMask stained cells, however, demonstrated a different percentage of "true spherocytes": $2.5 \%$ (A), $0 \%$ (B), and $0.08 \%$ (C). They are visualized in the dark colored boxes, each showing one cell from three perpendicular directions and mostly reflecting the amount observed in healthy subjects ( $0-0.3 \%$, examined in 15 donors). In contrast, many cells look like spherocytes from one direction (leftmost view in all boxes) but the other faces reveal different morphologies, such as mushroom-shaped cells, stomatocytes or other irregular-shaped cells (all light colored boxes) representing "pseudo spherocytes." These observations could be confirmed in 10 hereditary spherocytosis patients after 3D-imaging of about 1,000 cells per subject. This Figure is a reprint of Simionato et al. (2021).

mess depending on the reporting authors, hereditary dehydrated stomatocytosis (hDSt) is differentiated into Piezo- or Gárdosstomatocytosis, hDSt I and hDSt II or xerocytosis and Gárdos channelopathy, although this definition not always reflects the presence of stomatocytes or the dehydration state of the cell.

Also, in the case of hereditary spherocytosis we face two different problems: (i) sometimes spherocytes are not present and (ii) what we observe in blood films does not correspond to what we see in $3 \mathrm{D}$ (Figure 1). The $3 \mathrm{D}$ imaging approach helped us to realize that we need to think to a different nomenclature because morphology alone seems to be a limited diagnostic tool.

In any case, hereditary spherocytosis and hereditary stomatocytosis are presented here as examples to approach the problem. Hereditary spherocytosis because it is a well-known disease and among the rare anemias the most common one, with a clear diagnostic approach (Mohandas and Gallagher, 2008; Perrotta et al., 2008). However, in most centers of general medicine the first suspect of hereditary spherocytosis rises from the presence of spherocytes in the blood smear although it is known that the number of spherocytes may be low in most cases. On the contrary, the diagnosis of hereditary stomatosytosis was only based on morphological examination before the advent of the Next Generation Sequencing (NGS) technologies, and now we know that presence of stomatocytes may be associated to various different defects. Therefore, we are convinced the name "stomatocytosis" is an example that the nomenclature is, based on the current knowledge, an insufficient naming.

We admit that the naming is much less confusing when it comes to the enzymopathies, pyruvate kinase deficiency (Bianchi and Fermo, 2020) or G6PD deficiency (Luzzatto and Arese, 2018) name the defect protein.

We propose here to tackle the nomenclature of membranopathies by a holistic concept considering the entity of hereditary anemias and the genetic variants they are caused by. This initiative is meant to make a transition for the nomenclature of rare anemias. Although just for a few of them applies that "their names are just smoke and mirrors" the transition is meant to follow "Nomen est omen" for all of them.

\section{AUTHOR CONTRIBUTIONS}

All authors listed have made a substantial, direct and intellectual contribution to the work, and approved it for publication.

\section{FUNDING}

This study was supported by the European Framework Horizon 2020 under grant agreement number 860436 (EVIDENCE). 
Furthermore, we acknowledge support by the Deutsche Forschungsgemeinschaft (DFG, German Research Foundation) and Saarland University within the funding programme Open Access Publishing.

\section{REFERENCES}

Abay, A., Simionato, G., Chachanidze, R., Bogdanova, A., Hertz, L., Bianchi, P., et al. (2019). Glutaraldehyde - a subtle tool in the investigation of healthy and pathologic red blood cells. Front. Physiol. 10:514. doi: 10.3389/fphys.2019.00514

Adragna, N. C., Ferrell, C. M., Zhang, J., Fulvio, M. D., Temprana, C. F., Sharma, A., et al. (2006). Signal transduction mechanisms of $\mathrm{K}+-\mathrm{Cl}-$ cotransport regulation and relationship to disease. Acta Physiol. 187, 125-139. doi: 10.1111/j.1748-1716.2006.01560.x

Agre, P., Orringer, E. P., Chui, D. H., and Bennett, V. (1981). A molecular defect in two families with hemolytic poikilocytic anemia: reduction of high affinity membrane binding sites for ankyrin. J. Clin. Invest. 68, 1566-1576. doi: $10.1172 /$ JCI110411

Albuisson, J., Murthy, S. E., Bandell, M., Coste, B., Louis-dit-Picard, H., Mathur, J., et al. (2013). Dehydrated hereditary stomatocytosis linked to gain-of-function mutations in mechanically activated PIEZO1 ion channels. Nat. Commun. 4, 1-8. doi: $10.1038 /$ ncomms 3440

Andolfo, I., Alper, S. L., Franceschi, L. D., Auriemma, C., Russo, R., Falco, L. D., et al. (2013). Multiple clinical forms of dehydrated hereditary stomatocytosis arise from mutations in PIEZO1. Blood 121, 3925-3935. doi: 10.1182/blood-2013-02-482489

Andolfo, I., Russo, R., Gambale, A., and Iolascon, A. (2018). Hereditary stomatocytosis: an underdiagnosed condition. Am. J. Hematol. 93, 107-121. doi: 10.1002/ajh.24929

Andolfo, I., Russo, R., Manna, F., Shmukler, B. E., Gambale, A., Vitiello, G., et al. (2015). Novel Gardos channel mutations linked to dehydrated hereditary stomatocytosis (xerocytosis). Am. J. Hematol. 90, 921-926. doi: 10.1002/ajh.24117

Bamberg, E., and Passow, H. (1992) The Band 3 Proteins: Anion Transporters, Binding Proteins and Senescent Antigens. Amsterdam: Elsevier

Belkacemi, A., Trost, C. F., Tinschert, R., Flormann, D., Malihpour, M., Wagner, C., et al. (2021). The TRPV2 channel mediates Ca2+ influx and the D9THC-dependent decrease in osmotic fragility in red blood cells. Haematologica 106:274951. doi: 10.3324/haematol.2020.274951

Bessis, M. (1973). Living Blood Cells and Their Ultrastructure. Berlin, Heidelberg: Springer-Verlag.

Bessis, M. (1974). Corpuscles, Atlas of Red Blood Cell Shapes. Berlin, Heidelberg: Springer. doi: 10.1007/978-3-642-65657-6

Bianchi, P., and Fermo, E. (2020). Molecular heterogeneity of pyruvate kinase deficiency. Haematologica 105:241141. doi: 10.3324/haematol.2019.241141

Bianchi, P., Zaninoni, A., Fermo, E., Vercellati, C., Paola, M. A., Zanella, A., et al. (2015). Diagnostic power of laser assisted optical rotational cell analyzer (LoRRca MaxSis) evaluated in 118 patients affected by hereditary hemolytic anemias. Blood 126, 942-942. doi: 10.1182/blood.V126.23.942.942

Cisneros, G. S., and Thein, S. L. (2020). Recent advances in the treatment of sickle cell disease. Front. Physiol. 11:435. doi: 10.3389/fphys.2020.00435

Dagher, G., and Lew, V. L. (1988). Maximal calcium extrusion capacity and stoichiometry of the human red cell calcium pump. J. Physiol. 407, 569-586. doi: 10.1113/jphysiol.1988.sp017432

Fermo, E., Bogdanova, A., Petkova-Kirova, P., Zaninoni, A., Marcello, A. P., Makhro, A., et al. (2017). "Gardos Channelopathy": a variant of hereditary Stomatocytosis with complex molecular regulation. Sci. Rep. 7:1744. doi: 10.1038/s41598-017-01591-w

Fermo, E., Monedero-Alonso, D., Petkova-Kirova, P., Makhro, A., Pérès, L., Bouyer, G., et al. (2020). Gardos channelopathy: functional analysis of a novel KCNN4 variant. Blood Adv. 4, 6336-6341. doi: 10.1182 /bloodadvances.2020003285

Fischer, T. M. (2003). "Human red cell shape and the mechanical characteristics of the membrane," in Red Cell Membrane Transport in Health and Disease, eds I. Bernhardt and J. C. Ellory (Berlin, Heidelberg: Springer), 61-82. doi: 10.1007/978-3-662-05181-8_3

Glogowska, E., Lezon-Geyda, K., Maksimova, Y., Schulz, V. P., and Gallagher, P. G. (2015). Mutations in the Gardos channel (KCNN4) are associated with hereditary xerocytosis. Blood 126, 1281-1284. doi: 10.1182/blood-2015-07-657957

Huisjes, R., Makhro, A., Llaudet-Planas, E., Hertz, L., Petkova-Kirova, P., Verhagen, L. P., et al. (2019). Density, heterogeneity and deformability of red cells as markers of clinical severity in hereditary spherocytosis. Haematologica 105:188151. doi: 10.3324/haematol.2018.188151

Jarolim, P., Wichterle, H., Hanspal, M., Murray, J., Robin, H. L., and Palek, J. (1995). $\beta$ spectrinPRAGUE: a truncated $\beta$ spectrin producing spectrin deficiency, defective spectrin heterodimer self-association and a phenotype of spherocytic elliptocytosis. Brit. J. Haematol. 91, 502-510. doi: 10.1111/j.1365-2141.1995.tb05330.x

Kaestner, L., and Bianchi, P. (2020). Trends in the development of diagnostic tools for red blood cell-related diseases and anemias. Front. Physiol. 11:387. doi: 10.3389/fphys.2020.00387

Kaestner, L., Bogdanova, A., and Egee, S. (2020). Calcium channels and calciumregulated channels in human red blood cells. Adv. Exp. Med. Biol. 1131, 625-648. doi: 10.1007/978-3-030-12457-1_25

Khairy, K., Foo, J., and Howard, J. (2008). Shapes of red blood cells: comparison of 3D confocal images with the bilayer-couple model. Cell Mol. Bioeng. 1:173. doi: 10.1007/s12195-008-0019-5

Kihm, A., Kaestner, L., Wagner, C., and Quint, S. (2018). Classification of red blood cell shapes in flow using outlier tolerant machine learning. PLoS Comput. Biol. 14:e1006278. doi: 10.1371/journal.pcbi.1006278

Krishnevskaya, E., Payán-Pernía, S., Hernández-Rodríguez, I., Sevilla, Á. F. R., Serra, Á. A., Morales-Indiano, C., et al. (2021). Distinguishing iron deficiency from beta-thalassemia trait by new generation ektacytometry. Int. J. Lab Hematol. 43, e58-e60. doi: 10.1111/ijlh.13362

Lazarova, E., Gulbis, B., van Oirschot, B., and van Wijk, R. (2017). Next-generation osmotic gradient ektacytometry for the diagnosis of hereditary spherocytosis: interlaboratory method validation and experience. Clin. Chem. Lab. Med. 55, 394-402. doi: 10.1515/cclm-2016-0290

Lim, G., Wortis, M., and Mukhopadhyay, R. (2002). Stomatocyte-discocyteechinocyte sequence of the human red blood cell: evidence for the bilayercouple hypothesis from membrane mechanics. Proc. Natl. Acad. Sci. U. S. A. 99, 16766-16769. doi: 10.1073/pnas.202617299

Llaudet-Planas, E., Vives-Corrons, J. L., Rizzuto, V., Gómez-Ramírez, P., Navarro, J. S., Sibina, M. T. C., et al. (2018). Osmotic gradient ektacytometry: a valuable screening test for hereditary spherocytosis and other red blood cell membrane disorders. Int. J. Lab. Hematol. 40, 94-102. doi: 10.1111/ijlh.12746

Luzzatto, L., and Arese, P. (2018). Favism and glucose-6-phosphate dehydrogenase deficiency. New Engl. J. Med. 378, 60-71. doi: 10.1056/NEJMra1708111

Mohandas, N., and Gallagher, P. G. (2008). Red cell membrane: past, present, and future. Blood 112, 3939-3948. doi: 10.1182/blood-2008-07-161166

Mohandas, N., Lie-Injo, L., Friedman, M., and Mak, J. (1984). Rigid membranes of Malayan ovalocytes: a likely genetic barrier against malaria. Blood 63, 1385-1392. doi: 10.1182/blood.V63.6.1385.1385

Motulsky, A. G., Singer, K., Crosby, W. H., and Smith, V. (1954). The life span of the elliptocyte; hereditary elliptocytosis and its relationship to other familial hemolytic diseases. Blood 9, 57-72. doi: 10.1182/blood.V9.1.57.57

Pawley, J. B. (2006). Handbook of Biological Confocal Microscopy. Boston, MA: Springer Verlag. doi: 10.1007/978-0-387-45524-2

Peikert, K., Danek, A., and Hermann, A. (2017). Current state of knowledge in Chorea-Acanthocytosis as core Neuroacanthocytosis syndrome. Eur. J. Med. Genet. 61, 699-705. doi: 10.1016/j.ejmg.2017.12.007

Perrotta, S., Gallagher, P. G., and Mohandas, N. (2008). Hereditary spherocytosis. Lancet 372, 1411-1426. doi: 10.1016/S0140-6736(08)61588-3

Petrushanko, I., Yu., Mitkevich, V. A., Lakunina, V. A., Anashkina, A. A., Spirin, P. V., et al. (2017). Cysteine residues 244 and $458-459$ within the catalytic subunit of $\mathrm{Na}, \mathrm{K}$-ATPase control the enzyme's hydrolytic and signaling function under hypoxic conditions. Redox. Biol. 13, 310-319. doi: 10.1016/j.redox.2017.05.021

Quint, S., Christ, A. F., Guckenberger, A., Himbert, S., Kaestner, L., Gekle, S., et al. (2017). 3D tomography of cells in micro-channels. Appl. Phys. Lett. 111:103701. doi: $10.1063 / 1.4986392$ 
Rapetti-Mauss, R., Lacoste, C., Picard, V., Guitton, C., Lombard, E., Loosveld, M., et al. (2015). A mutation in the Gardos channel is associated with hereditary xerocytosis. Blood 126, 1273-1280. doi: 10.1182/blood-2015-04-642496

Rotordam, G. M., Fermo, E., Becker, N., Barcellini, W., Brüggemann, A., Fertig, N., et al. (2018). A novel gain-of-function mutation of Piezol is functionally affirmed in red blood cells by high-throughput patch clamp. Haematologica 104:e179-83. doi: 10.3324/haematol.2018.201160

Shmukler, B. E., Vandorpe, D. H., Rivera, A., Auerbach, M., Brugnara, C., and Alper, S. L. (2014). Dehydrated stomatocytic anemia due to the heterozygous mutation R2456H in the mechanosensitive cation channel PIEZO1: a case report. Blood Cells Mol. Dis. 52, 53-54. doi: 10.1016/j.bcmd.2013.07.015

Simionato, G., Hinkelmann, K., Chachanidze, R., Bianchi, P., Fermo, E., Wijk, R., et al. (2021). Red blood cell phenotyping from 3D confocal images using artificial neural networks. PLoS Comput. Biol. 17:e1008934. doi: 10.1371/journal.pcbi.1008934

Soderquist, C., and Bagg, A. (2013). Hereditary elliptocytosis. Blood 121, 3066-3066. doi: 10.1182/blood-2012-09-457788

Vives-Corrons, J.-L., Krishnevskaya, E., Rodriguez, I. H., and Ancochea, A. (2021). Characterization of hereditary red blood cell membranopathies using combined targeted next-generation sequencing and osmotic gradient ektacytometry. Int. J. Hematol. 113, 163-174. doi: 10.1007/s12185-020-03010-9

Walker, R. H., and Danek, A. (2021). "Neuroacanthocytosis" - overdue for a taxonomic update. Tremor Other Hyperkinet. Mov. 11:1. doi: 10.5334/tohm.583
Wenk, R. E. (1976). Comparison of five methods for preparing blood smears. Am. J. Med. Technol. 42, 71-78.

Zaninoni, A., Fermo, E., Vercellati, C., Consonni, D., Marcello, A. P., Zanella, A., et al. (2018). Use of laser assisted optical rotational cell analyzer (LoRRca MaxSis) in the diagnosis of rbc membrane disorders, enzyme defects, and congenital dyserythropoietic anemias: a monocentric study on 202 patients. Front. Physiol. 9:451. doi: 10.3389/fphys.2018.00451

Zarychanski, R., Schulz, V. P., Houston, B. L., Maksimova, Y., Houston, D. S., Smith, B., et al. (2012). Mutations in the mechanotransduction protein PIEZO1 are associated with hereditary xerocytosis. Blood 120, 1908-1915. doi: 10.1182/blood-2012-04-422253

Conflict of Interest: The authors declare that the research was conducted in the absence of any commercial or financial relationships that could be construed as a potential conflict of interest.

Copyright (c) 2021 Simionato, van Wijk, Quint, Wagner, Bianchi and Kaestner. This is an open-access article distributed under the terms of the Creative Commons Attribution License (CC BY). The use, distribution or reproduction in other forums is permitted, provided the original author(s) and the copyright owner(s) are credited and that the original publication in this journal is cited, in accordance with accepted academic practice. No use, distribution or reproduction is permitted which does not comply with these terms. 